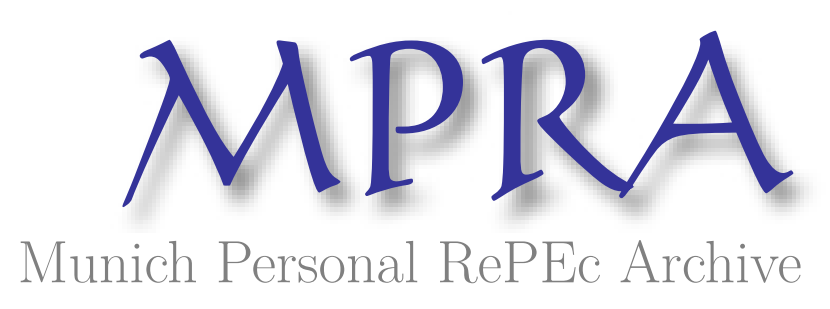

\title{
Heritability of time preference: Evidence from German twin data
}

Hübler, Philipp

15 March 2017

Online at https://mpra.ub.uni-muenchen.de/77620/

MPRA Paper No. 77620, posted 18 Mar 2017 11:48 UTC 


\title{
Heritability of time preference: Evidence from German twin data
}

- Preliminary, please do not cite or circulate without the author's permission -

\author{
Philipp Hübler*
}

15th March 2017

\begin{abstract}
Intergenerational correlations of time preference are well documented. However, there is still limited empirical evidence about the role of genetics in this transmission process. In our paper, we use data on roughly 3,000 twins from the German TwinLife project to estimate the heritability of time preference. We rely on an experimentally validated survey measure of temporal discounting, namely, self-assessed patience. The analysis of monozygotic and dizygotic twins enables us to apply standard biometric models. We find that genetic differences explain up to 23 percent of individual variation in patience. Whereas the additive genetic effect and common environmental effects are of minor importance, a major dominant genetic effect is present. These results indicate a notable degree of genetic influence on economic time preferences.
\end{abstract}

Keywords: Twin study; genetics; heritability; patience; time preference.

*University of Augsburg, Department of Economics, Universitätsstr. 16, 86159 Augsburg, Germany. E-mail: philipp.huebler@wiwi.uni-augsburg.de, Phone: +49-821-598-4209, Fax: +49-821-598-4232. 


\section{Introduction}

In economics, individual preferences are highly important since they affect people's decision-making. Next to the exhaustively studied role of risk preferences, the economic concept of time preference is particularly relevant. Time preferences are significantly involved in a person's intertemporal choices. In general, they influence decisions that include a trade-off between costs today and (potential) benefits in the future (see Frederick et al. 2002 for an excellent review of the concept of time discounting). Time preferences are related to a variety of crucial lifetime outcomes such as educational attainment and personal income. For instance, Golsteyn et al. (2014) show that patience predicts success in school as well as higher earnings in the long run. In addition, present-biased individuals are less likely to save money for the future. In contrast, they are more likely to run into debt (Meier and Sprenger 2010). Moreover, a high discount rate (low level of patience) is associated with a number of adverse health behaviors and outcomes such as smoking (Kang and Ikeda 2014), frequent alcohol consumption (Rossow 2008) and obesity (Komlos et al. 2004). ${ }^{1}$

In recent years, the empirical evidence on the intergenerational transmission of time preference has increased. For instance, Gauly (2016) finds a positive correlation between parental and offspring time preference using representative household survey data from Germany. Other studies show similar results. ${ }^{2}$ Despite all these findings, a fundamental question remains unanswered. How are time preferences formed? Apparently, time preferences differ across individuals (e.g., Barsky et al. 1997). But where does this variation come from? Motivated by psychologists, the nature-nurture debate is concerned with the decomposition of genetic and environmental influences on human traits or phenotypes. "Nature" refers to the relative contribution made by genetic inheritance and related biological factors. "Nurture" represents the influence that comes from external sources such as parents' socialization efforts, imitation/learning or unique experiences. In fact, previous studies found that both, inherited and acquired characteristics, determine behavioral traits and/or outcomes (see e.g., Björklund et al. 2007).

In this paper, we want to shed light on the fraction of variance in time preference that is explained by genetics. Relying on twin data from Germany, the comparison of identical/monozygotic (MZ) and fraternal/dizygotic (DZ) twins enables us to estimate the proportion of variation in individual time preference that is due to genetic and environmental factors. Both types of twins are considered to share the same (family) environment, but fundamental differences in genetic relatedness exist between them.

\footnotetext{
${ }^{1}$ Time preference, time discounting, discount rate, patience and future orientation are used as synonyms throughout the paper.

${ }^{2}$ See, for example, Brown and van der Pol (2015), Arrondel (2013), Kosse and Pfeiffer (2012), Gouskova et al. (2010), Reynolds et al. (2009), Webley and Nyhus (2006) and Knowles and Postlewaite (2005).
} 
Thus, any excess similarity of MZ twin pairs compared to DZ twin pairs with respect to a specific phenotype indicates the presence of genetic effects. Studies simply analyzing the raw intergenerational correlation of a phenotype are not able to decompose this variation adequately.

Evidence on the heritability of time preference is scarce. To the best of our knowledge, there have been two studies addressing a similar research question. Anokhin et al. (2011) analyzed the heritability of delay discounting in a longitudinal twin design. Using a delay of gratification method, they find that genetic factors contribute $30 \%$ and $51 \%$ to the variation in delay discounting at ages 12 and 14, respectively. Cronqvist and Siegel (2015) rely on a set of MZ and DZ twins from the Swedish Twin Registry (STR). They apply a person's saving behavior as a proxy for individual time preference. They show that genetic differences explain roughly one third of the total variation in savings propensities across individuals. Interestingly, much more evidence exists with respect to the genetic variation in economic risk preferences. The relative contribution of genetics to risk attitudes varies considerably across studies. Whereas Cesarini et al. (2009) show that heritability is around 20\%, Zhong et al. (2009) and Zyphur et al. (2009) find magnitudes of $57 \%$ and $63 \%$, respectively.

In line with the previous literature, our paper employs the classic twin methodology. However, we contribute to the literature in several ways. We are the first to use a novel twin data set from Germany to estimate the genetic variation in time preference. Crosssectional data is available for adolescents and young adults between the ages of 10-25. In comparison to the vast majority of twin studies, the sample size is large. Our final sample contains information on roughly 3,000 twins. Moreover, we use a direct survey measure of time preference. A question on the general patience of an individual acts as a proxy for time preference. An incentivized experiment showed that self-assessed patience is a meaningful proxy for time preference as elicited using the typical price list decision format (Vischer et al. 2013). The general level of patience is likely to evaluate a person's time preference more comprehensively than relying on her savings behavior. The latter represents an outcome of time discounting rather than a direct measurement of time preference.

Our empirical analysis provides evidence that time preferences are partly heritable. According to our best-fitting model, we find that differences in genetics explain about 23 percent of the variation in patience across individuals. The estimates of heritability are primarily driven by a non-additive (dominant) genetic effect. A negligible influence of the twins' shared environment is identified. The remainder of the paper is organized as follows. Section 2 describes the twin data and the measurement of time preference. Section 3 provides information on the basic twin methodology and the statistical analysis. Results are presented in Section 4. Finally, Section 5 concludes with a discussion of the main findings. 


\section{Data}

\subsection{Twin data}

We use novel twin data from the German TwinLife project. The main purpose of this twin family study is to improve the understanding of the development of social inequalities over the life course. Next to its longitudinal design, TwinLife combines a multi-cohort cross-sequential and an extended twin family design. It observes four birth cohorts of MZ and DZ twins over a 12-year period. Twins born in the years 1990-1993, 1997/1998, 2003/2004 and 2009/2010 as well as their parents and twins' siblings (if available) are included. The project began in 2014 and is supposed to end with the last survey wave in 2023. Interviews are conducted on a yearly basis. Overall, this twin survey will provide data of a representative sample of about 4,000 German twin families. Currently, data on the first household interview for the first partial wave on 2,009 twin families are available. In order to prevent distortions due to gender differences, only twin pairs of the same sex are surveyed. Furthermore, only such twins are examined that have grown up or are still growing up in the same family. More details on the conception and design of the TwinLife study can be found elsewhere (Hahn et al. 2016). ${ }^{3}$

For the emprirical analysis, we exclude all twins from the youngest birth cohort. Twins born in 2009/2010 were about five years old during the first survey wave in 2014 and therefore too young to answer the survey question on time preference. Further restrictions were necessary, since not all individuals provided information on time preference. Hence, we exclude all respondents with missing information. The remaining twins are adolescents and young adults aged 10-25 years. Basically, all three cohorts comprise a similar number of twins. The first cohort of twins contains 990 twins aged 10-12, the second comprises 1034 twins aged 16-18 and the third includes 932 twins aged 22-25. It allows us to cover a great age range. This time span is of particular interest since it clusters decisions on education, employment, health, etc. where time preferences are always involved. All these decisions made will affect lifetime outcomes (e.g., income) in the future.

The zygosity of twins was verified with a specific zygosity questionnaire. In addition, all results obtained from the standard questionnaire items where validated with a saliva test. This DNA test is a standard procedure to tell if the twins are monozygotic or dizygotic and is generally considered to be highly reliable. Our final sample contains information on $703 \mathrm{MZ}$ twin pairs (1406 twins) and $775 \mathrm{DZ}$ twin pairs (1550 twins). Hence, the total sample size sums up to 2956 individuals. Table 1 shows the number of twins separated by zygosity and sex. Opposite-sexed dizygotic twins are not surveyed.

\footnotetext{
${ }^{3}$ Additional information is also available online: http://www.twin-life.de/en.
} 
[Insert Table 1 about here.]

\subsection{Measuring time preference}

The twin questionnaire contains a suitable survey question to elicit individual time discounting. Each respondent has to rate his or her personal level of patience according to a 11-point scale. The exact wording of the question is as follows: "How would you describe yourself: Are you generally an impatient person, or someone who always shows great patience? Please tick a box on the scale, where the value 0 means: "very impatient" and the value 10 means: "very patient". You can use the values in between to make your estimate." Hence, self-reported patience is used as a proxy for individual time preference. In our sample, the average patience level is 5.75 .

The behavioral relevance of our time preference measure has been explicitly validated. This ultra-short survey measure of patience was first introduced in the 2008 questionnaire of the German Socio-Economic Panel (SOEP). The SOEP is an annual panel survey conducted since 1984. Each year more than 20,000 individuals $(12,000$ households) representative of the German population are surveyed (see Wagner et al. 2007). Vischer et al. (2013) conducted an incentivized experiment with 977 participants forming a representative sub-sample of the adult population to the 2006 wave of the SOEP. Subjects were asked to indicate their preferences in a choice over a 12-month time horizon. ${ }^{4}$ The results show that those who rank themselves as 'more impatient' in the survey in 2008 also exhibit a higher degree of impatience in the experiment in 2006. Hence, this simple and ultra-short survey measure of patience turns out to be a meaningful proxy for time preference. These findings remain robust even after controlling for impulsivity. ${ }^{5}$ Indeed, this demonstrates that the question on general patience is unambiguous. Thus, a respondent's misinterpretation of the more future-oriented aspects underlying the general question about patience can be ruled out. Table 2 reports some summary statistics of our twin sample. Apparently, Figure 1 indicates that variation in self-assessed patience is present across individuals. ${ }^{6}$

\footnotetext{
${ }^{4}$ In the experiment, choice tables with the typical price list decision format were used. The participants had to declare their preferences by choosing between an immediate (left column) or delayed payment (right column). The immediate payment was continuously fixed (€200). However, the delayed payment varied in each of the 20 choice situations and increased by 2.5 percentage points (compounded semi-annually) from row to row. Switching from left to right (and sticking to the delayed payment in all subsequent rows) indicates the bounds of the discount rate the respondent claims in order to wait for pay-out an additional time period of 12 months. Before the start of the experiment, the participants were informed that one of their choices would be randomly selected for payment. In the second random step one out of nine participants were actually paid by cheque according to the previous choice.

${ }^{5}$ In addition to impulsivity, including a control variable for personal risk attitudes does not affect the results either.

${ }^{6}$ Throughout the empirical analysis, we use the standardized version of patience.
} 
[Insert Table 2 about here.]

[Insert Figure 1 about here.]

\section{Twin methodology}

The classic twin design contains information on both, monozygotic (MZ) and dizygotic (DZ) twins. This enables us to partition the observed variance in a trait (e.g., patience) into genetic and environmental components. Overall, four latent factors can be taken into account. According to the polygenic model proposed by Fisher (1918), the genetic variation in a phenotype can be divided into two components. The additive genetic effect (A) describes the influence of different alleles which are added up when being passed from the parents to the offspring. The second genetic component consists of non-additive/dominant genetic effects (D). These effects are characterized by allelic interactions within genes. Hence, these genetic influences are transmitted from parents to their offspring in a dominant/recessive way.

With respect to the environmental components, shared and non-shared environmental effects can be distinguished. The common environment component $(\mathrm{C})$ captures all influences shared by the twins reared in the same family. Thus, both twins of a twin pair are equally affected. This includes the same prenatal environment, home environment (e.g., parenting style), socioeconomic status of the parents/family, and the like. On the contrary, the unique environmental effects (E) are not shared by the twins. They are different across the twins of a twin pair. These individual-specific influences refer to unique experiences with friends, sexual partners, illness or injury, and the like.

Based on this logic, the standard twin model decomposes the total variance of patience into A, D, C and E components. The corresponding ADCE model for individual $j$ in family $i$ can be written as an error components model

$$
P_{a t}=\mu+A_{i j}+D_{i j}+C_{i}+E_{i j}
$$

where $\mu$ is the overall mean, $A_{i j} \sim \mathrm{N}\left(0, \sigma_{A}^{2}\right)$ is the additive genetic component, $D_{i j} \sim$ $\mathrm{N}\left(0, \sigma_{D}^{2}\right)$ is the dominant genetic component, $C_{i} \sim \mathrm{N}\left(0, \sigma_{C}^{2}\right)$ is the common environental component, and $E_{i j} \sim \mathrm{N}\left(0, \sigma_{E}^{2}\right)$ is the non-shared environmental component. ${ }^{7}$ Thus, the total variance of patience is represented by the sum of the four mutually independent variance components

$$
\operatorname{Var}\left(P a t_{i j}\right)=\sigma_{A}^{2}+\sigma_{D}^{2}+\sigma_{C}^{2}+\sigma_{E}^{2}
$$

\footnotetext{
${ }^{7}$ For notational convenience, the random error term $\epsilon_{i j}$ is replaced by $E_{i j}$ in Equation (1). The error term is uncorrelated across twins and therefore included in the non-shared environment component.
} 
Genetic theory shows that differences in the genetic relatedness between MZ and DZ twins exist (see Neale and Maes 2004). MZ twins develop from the splitting of the same fertilized egg into two, whereas DZ twins develop from two different eggs fertilized by two different sperm cells. Hence, MZ twins share all their genes. On the contrary, DZ twins share (on average) only half their genes. Among DZ twins, this results in a correlation of 0.5 for additive genetic effects and a correlation of 0.25 for dominant genetic effects. Since the shared environment component is reasonably assumed to be the same for both members of a twin pair, the correlation is perfect (1.0) across twin pairs, regardless of zygosity. The non-shared environmental component is unique to each twin and therefore completely uncorrelated across twins, regardless of zygosity. Due to these genetic differences, the variance-covariance matrix varies by type of twin pair

$$
\begin{gathered}
\sum_{P a t}^{M Z}=\left(\begin{array}{cc}
\sigma_{A}^{2} & \sigma_{A}^{2} \\
\sigma_{A}^{2} & \sigma_{A}^{2}
\end{array}\right)+\left(\begin{array}{cc}
\sigma_{D}^{2} & \sigma_{D}^{2} \\
\sigma_{D}^{2} & \sigma_{D}^{2}
\end{array}\right)+\left(\begin{array}{cc}
\sigma_{C}^{2} & \sigma_{C}^{2} \\
\sigma_{C}^{2} & \sigma_{C}^{2}
\end{array}\right)+\left(\begin{array}{cc}
\sigma_{E}^{2} & 0 \\
0 & \sigma_{E}^{2}
\end{array}\right) \\
\sum_{P a t}^{D Z}=\left(\begin{array}{cc}
\sigma_{A}^{2} & 0.5 \sigma_{A}^{2} \\
0.5 \sigma_{A}^{2} & \sigma_{A}^{2}
\end{array}\right)+\left(\begin{array}{cc}
\sigma_{D}^{2} & 0.25 \sigma_{D}^{2} \\
0.25 \sigma_{D}^{2} & \sigma_{D}^{2}
\end{array}\right)+\left(\begin{array}{cc}
\sigma_{C}^{2} & \sigma_{C}^{2} \\
\sigma_{C}^{2} & \sigma_{C}^{2}
\end{array}\right)+\left(\begin{array}{cc}
\sigma_{E}^{2} & 0 \\
0 & \sigma_{E}^{2}
\end{array}\right)
\end{gathered}
$$

where the variances of the twins are situated in the diagonal and the corresponding covariance between twin 1 and twin 2 is situated in the off-diagonal of each matrix. ${ }^{8}$ Differences in the covariances between MZ and DZ twins point towards the importance of genetics. Similarities in the covariance structure across twin pairs with different zygosity can be attributed to the shared environmental component.

The estimate of interest is the (broad) heritability coefficient which can be written as

$$
H_{\text {Pat }}=\frac{\sigma_{A}^{2}+\sigma_{D}^{2}}{\sigma_{A}^{2}+\sigma_{D}^{2}+\sigma_{C}^{2}+\sigma_{E}^{2}}
$$

It gives the degree to which genetics contribute to the total variation in patience across individuals.

The polygenic model is estimated under the standard assumptions of biometric modeling. MZ twins are considered to be genetically identical and the equal environment assumption holds true for MZ and DZ twins. No gene-environment correlations or interactions are present. Another non-technical assumption is the absence of non-random

\footnotetext{
${ }^{8}$ It is further assumed that the MZ and DZ twin pairs are unrelated. The general notation of a variance-covariance matrix in the twin context is

$$
\sum=\left(\begin{array}{cc}
\text { variance of first twin } & \text { covariance of twins } \\
\text { covariance of twins } & \text { variance of second twin }
\end{array}\right)
$$
}


pairing of the twins' parents. The most important technical assumptions are equal mean and variance of twin 1 and twin 2 and MZ and DZ twins. This is required to estimate the model since the same path coefficients are applied for both, MZ and DZ twins. Moreover, the maximum likelihood method is used for estimation and inference. It assumes bivariate normality of the paired observations.

A disadvantage of this biometric analysis is that it is impossible to estimate all four components of the ADCE model simultaneously with classic twin data alone. Especially the components $\mathrm{D}$ and $\mathrm{C}$ make opposite predictions about the relative difference between $\mathrm{MZ}$ and DZ correlations. Hence, both $\mathrm{C}$ and D are confounded in a classic twin study. Common environmental influences make DZ correlations more similar to MZ correlations. However, the presence of dominant genetic effects makes DZ correlations less similar to MZ correlations. The primary reason for that is that D correlates perfectly for MZ twin pairs whereas the correlation is only 0.25 for DZ twin pairs. Therefore, in practice, $\mathrm{ACE}$ or $\mathrm{ADE}$ models and their respective submodels (e.g., $\mathrm{AE}, \mathrm{DE}$ or $\mathrm{CE}$ models) are estimated. Which model to use highly depends on the information retrieved from the twin data. Genetic heuristics predict the existence of genetic effects when the trait correlation among MZ twin pairs is higher than the correlation among DZ twin pairs. For instance, a correlation among MZ twin pairs that is more than two times larger than the corresponding correlation among DZ twin pairs is a strong indicator for a prominent genetic dominance effect (D). In this case, the influence of the shared environment component $(\mathrm{C})$ is likely to be negligible and the estimation of an ADE model would be recommended (Neale and Maes 2004).

To provide evidence on the heritability of time preference/patience, we follow three basic steps. First, we start with a mean comparison across MZ and DZ twin pairs. Second, we compare the intraclass correlation coefficients across both twin types. This should provide us a guideline for the biometric analysis. Finally, we apply maximumlikelihood based structural equation modeling to elicit the best-fitting polygenic model and report the relative contributions of the respective variance components. The statistical tool (twinlm) in the Analysis of Multivariate Events mets-package in R is used to conduct the structural equation analysis (Holst and Scheike 2017). ${ }^{9}$

\footnotetext{
${ }^{9}$ As a robustness check, we re-run the biometrical analysis in Stata (version 12.1) according to the twin model parameterization proposed by Rabe-Hesketh et al. (2008). We obtain qualitatively similar results to those presented in the upcoming Section 4.
} 


\section{Results}

We start with reporting basic statistics on patience for MZ and DZ twins. In Figure 2, we plot a histogram of the distribution for patience, separately, for MZ and DZ twins. In general, identical twins seem to be more patient than fraternal twins. This initial finding is supported by the comparison of the average level of patience between both twin types. The mean levels of patience are reported in Table 3.

\section{[Insert Figure 2 about here.]}

[Insert Table 3 about here.]

On average, MZ twins report a general level of patience of about 5.9. DZ twins are slightly more impatient. The corresponding level of patience is roughly 5.6. However, the difference between means is highly significant. Testing the equality of means rejects the null hypothesis of equal means at the $1 \%$ level. In Table 4, we report the MZ and DZ twin pair correlations of patience. Spearman's correlation coefficient for MZ twin pairs is 0.241 and highly significant. The respective correlation for DZ twin pairs is basically zero. ${ }^{10}$ These findings provide first evidence that genetics seem to play a role in the variance of individual patience. The substantial difference in MZ and DZ correlations can also be considered graphically. In Figure 3, we plot twin's patience against co-twin's patience. It supports the calculated correlations from above.

[Insert Table 4 about here.]

[Insert Figure 3 about here.]

In the following, we present our findings from the biometric analyses. The results from the model selection process and from the variance component analysis are shown in Table 5. ${ }^{11}$ All models include control variables for age and sex. In order to appropriately test the overall fit of each polygenic model, we use the saturated model as a benchmark. The saturated model provides the means, variances and covariances without imposing restrictions. Hence, it is the least restrictive model since no constraints/technical assumptions are made. Comparing the genetic twin models (e.g., the ADE model) to the

\footnotetext{
${ }^{10}$ The Spearman correlations are very similar to the Pearson correlations. Pearson's correlation for MZ twin pairs is $0.233(\mathrm{p}<.01)$. Pearson's correlation for DZ twin pairs is 0.021 and not statistically significant.

${ }^{11}$ Although we fully recognize the ordinal measurement of our time preference proxy, we treat selfreported patience as a continuous variable. Cesarini et al. (2009) have shown that the polygenic models yield similar results, regardless of whether the dependent variable is treated as continuous or ordinal.
} 
saturated model allows to evaluate the actual model fit. If the main assumptions are fulfilled, no significant drop in model fit should be observed.

We decide to test the ADE model against the saturated model. ${ }^{12}$ The existing differences in correlation between the MZ and DZ twin pairs (0.234 vs. 0.006) suggest the presence of a dominant genetic effect on the variance in patience. Hence, twin theory suggests that a standard ACE model would be inappropriate. According to Plomin et al. (2013), dominant gegentic effects preclude an influence of the common environment. Here, we fail to reject the hypothesis that the saturated model has a better fit than the ADE model $(\mathrm{p}<.05)$. However, this occurs frequently in twin studies with a (very) large sample size. Even minor differences in variances between twin groups can be highly significant (see e.g., Waszczuk et al. 2015). Moreover, in fairly large twin samples the consideration of the BIC criterion is advised. It takes into account the underlying sample size. The BIC criterion is considerably smaller for the ADE model, indicating a better fit than the saturated model (see e.g., Dale et al. 2015).

According to the Likelihood ratio test, dropping the $\mathrm{D}$ component from the ADE model significantly reduces model fit $(\mathrm{LRT}=23.499, \mathrm{p}<.01)$. Eliminating the additive genetic component (A) from the ADE model does not worsen the model fit (LRT=6.077, $\mathrm{p}>.10) .{ }^{13}$ This is not surprising because $\mathrm{A}$ is estimated zero in the ADE model. These findings suggest that dominant genetic effects are present. On the contrary, the additive genetic component is negligible. Hence, we select the DE model as our preferred polygenic model. According to the Likelihood ratio test, the submodel does not perform sigificantly worse than the ADE model. Moreover, it shows a slighly better fit according to the standard criteria. In general, a lower AIC/BIC indicates better model fit. Therefore, the results of the DE model indicate a heritability of patience of roughly one quarter. In other words, we find that differences in genetics account for 23 percent of the overall variation in general patience across individuals. ${ }^{14}$ Additive genetic effects as well as shared environmental effects are negligible. Consequently, the largest proportion of variance is attributed to unique environmental influences (E). They account for approximately three quarters of the total variation in patience. However, it is important to keep in mind that the error term is also included in E.

[Insert Table 5 about here.]

\footnotetext{
${ }^{12}$ All of the theoretical twin models represent submodels of the saturated model.

${ }^{13}$ The original p-values obtained from the Likelihood ratio tests comparing the ADE model to the AE and DE models were too conservative. According to Dominicus et al. (2006), we made the recommended adjustments and calculated the appropriate values.

${ }^{14}$ Zyphur et al. (2009) argue that it is generally inappropriate to choose the DE model over the ADE model even though the A component is virtually zero. However, the estimate of heritability is the same, whether relying on the ADE or the more parsimonious DE model.
} 
Before we further discuss our findings, we would like to make some comments on the most important assumptions underlying the polygenic models from above. First, the equal environment assumption states that environmentally caused similarity is the same for MZ and DZ twin pairs. This sounds plausible since both twin types share the womb at the same time, are the same age and are raised together in the same family environment. However, this assumption is not free of criticism. For instance, Plomin et al. (2013) provide some evidence that MZ twins are likely to be treated more similarly by their parents than DZ twins. However, research has shown that any potential bias due to violations of the equal environment assumption is not of first order importance (Bouchard 1998). Moreover, Bouchard et al. (1990) estimated similar heritability coefficients, regardless of whether using twins reared together or apart. Hence, heritability bias arising from equal environment violations are negligible.

Second, we test the assumption of random mating of mothers and fathers. In comparison to the vast majority of twin studies, we have the possibility to analyze the correlation of patience between parents. The parents of the twins were asked to rate their level of patience on the 11-point scale as well. We merged their answers with the corresponding answers provided by the twins. Unfortunately, only two-third of the fathers in the survey has reported non-missing information on patience. By contrast, almost all surveyed mothers - probably because of their role as primary caregiver - have indicated their general level of patience. Inevitably, this circumstance reduces the actual sample size to roughly 2,000 observations and therefore differs from the number of observations used for the classic biometrical analyis. However, the results point towards random mating. We find a negative correlation between maternal and paternal patience that is not statistically significant $(\rho=-0.03, \mathrm{p}>.10)$. In general, there is very limited evidence on assortative mating regarding time preference. To the best of our knowledge, Gauly (2016) is the only study explicitly addressing this issue. Her evidence suggests a weakly significant correlation which is also negative. Hence, further investigations of assortative mating in the field of time discounting seems to be a promising area for future research.

In general, standard twin models assume the lack of any gene-environment interactions. In fact, it is (almost) impossible to provide conclusive evidence that this assumption holds completely true. To test this assumption properly, you would need to have a dataset incredibly rich in information. Depending on the subject under investigation, you may require longitudinal trait data, information on non-adoptees and adoptees or explicit environmental measures (see Rijsdijk and Sham (2002) for a critical discussion of the underlying assumptions). However, as it is common practice in the twin literature, we assume that the absence of gene-environment interactions holds true. All in all, we are confident that our biometrical analyses are not biased due to fundamental violations of the standard assumptions of the twin method. 


\section{Discussion and conclusion}

The main purpose of this paper was to provide evidence on the general formation of time preference. In particular, we analyzed the degree to which time preferences are heritable. We rely on novel twin data from the German TwinLife project. It is the first large-scale twin study in Germany. We use self-reported patience as a meaningful proxy for individual time discounting. Standard biometric analyses are conducted. According to the model selection process, the DE model gives the best fit. In this model, the dominant genetic effect is estimated to 23 percent. Hence, almost one quarter of the total variance in patience can be attributed to genetic influences.

Unlike the findings from Anokhin et al. (2011) and Cronqvist and Siegel (2015), this is the first paper that reveals a substantial contribution of the dominant genetic component (D) to the overall variation in time preference. For instance, with respect to the genetics of risk preferences, Zyphur et al. (2009) and Zhong et al. (2009) find similar results. Both conclude that the attitude towards risk is a non-additive trait which is genetically coded in a dominant/recessive way.

Inevitably, this raises the question about candidate genes which are related to time discounting. In recent years, molecular genetics has spared no efforts to disentangle the complex genetic architecture of human personality. For example, Eisenberg at al. (2007) and Carpenter et al. (2011) found that the Dopamine Receptor D4 gene (DRD4) predicts impulsivity and time preference, respectively. Furthermore, links between serotonin (5-hydroxytryptamine, 5-HT) and personality traits such as impulsivity have been established (e.g., Carver and Miller (2006), Oades et al. (2008) and Miyazaki et al. (2012)). We have to admit that most of these studies explicitly focus on impulsivity and not so much on (long-term) time preference/patience. However, although impulsivity is a slightly different concept in the context of time discounting, it is directly related to patience (see Kalenscher and Pennartz (2008) for an extensive review of the neuroeconomics of intertemporal decision-making). For example, Gauly (2016) shows a significant negative intrapersonal correlation between general patience and impulsiveness. Overall, there is some evidence that dominant/recessive genes account for differences in serotonin and/or dopamin levels that are further related time discounting. But more research is needed to provide more conclusive evidence for the nonadditivity in the genetics of time preference.

However, our study has some limitations. Unfortunately, we are not able to exploit the longitudinal design of the TwinLife project. While writing these lines, only data on the first partial wave with information on time preference was available for researchers. However, for future research, it seems to be promising to expand the cross-sectional analysis with panel data. This would allow to analyze possible changes in the degree of heritability over the twins' lifetime. 
Due to the basic concept of the TwinLife study, we only have data on same-sex twin pairs. Since MZ twin pairs are always the same sex by nature, DZ twins could differ. However, information on DZ twin pairs consisting of a female and male individual is not available in this twin study. Opposite-sex DZ twin pairs would have allowed to conduct further investigations of the fairly low DZ correlations.

A further limitation is that the survey question on general patience is a rather universal measure of a person's time preference. But we should be aware of the possibility that time preferences may be domain-specific (Tsukayama and Duckworth 2010). Thus, the degree of heritability of time preference might vary across different domains (e.g., monetary vs. health domain). For instance, Bickel et al. (1999) show that discount rates are higher in the health domain than in the money domain. Future research is highly encouraged to dive into the genetics of time preferences in different domains.

Yet another shortcoming is that we cannot completely rule out misreporting among children. Our youngest cohort consists of twins aged 10-12 years. The survey question on general patience is measured on a standard 11-point scale for all respondents. Although the wording of the question is age-adjusted for this specific cohort, some children may have experienced difficulties in answering this question appropriately (Mellor and Moore 2014). Thinking about their own personality and providing the corresponding answer on a Likert scale requires a sufficiently high level of abstract thinking of those children. Thus, it is likely that at least some twins of this particlar birth cohort have not fully developed their abstract thinking skills at this stage of life (Maćkiewicz and Cieciuch 2016). However, we are confident that potential measurement bias is negligible. We excluded all twins falling into the respective age range and re-estimated the corresponding polygenic models. Although we lose a substantial number of observations (one third of the sample size), we still obtain a heritability coefficient of around 20 percent. Moreover, using a uniform measurement of patience for all respondents ensures consistency and comparability across birth cohorts.

Despite the limitations mentioned above, we conclude that our findings provide solid evidence that time preferences are broadly heritable. However, the majority of variation in patience can be attributed to (unique) environmental factors. From a policy perspective, this is good news. We infer that people can be affected by public policy interventions that intend to make them more future-oriented. For instance, a trait that is fully heritable (100\%) would leave no scope for any interfering actions. Such interventions may include information campaigns about the adverse health consequences of smoking, a proper design of commitment devices to save more money for the future or school-subsidies to show children that today's investments in education will result in higher earnings in the (distant) future. Hence, our results indicate that public policy interventions with the aim of nudging people towards more future orientation and away from rather seductive and elusive instant gratification may have a good prospect of 
success. Since the literature on the genetics of time preferences is scarce, we want to encourage future research to dedicate more time on the investigation of the genetics of time discounting. However, it is already fair to say that the role of genetics in intertemporal decision-making should no longer be ingnored. 


\section{References}

Anokhin, A. P.; S. Golosheykin, J. D. Grant and A. C. Heath (2011): Heritability of delay discounting in adolescence: a longitudinal twin study. Behavior Genetics, 41(2): 175-183.

Arrondel, L. (2013): Are "daddy's boys" just as rich as daddy? The transmission of values between generations. The Journal of Economic Inequality, 11(4): 439-471.

Barsky, R. B.; M. S. Kimball, F. T. Juster and M. D. Shapiro (1997): Preference parameters and behavioral heterogeneity: An experimental approach in the health and retirement study. Quaterly Journal of Economics, 112: 537-579.

Bickel, W. K.; A. L. Odum and G. J. Madden (1999): Impulsivity and cigarette smoking: delay discounting in current, never, and ex-smokers. Psychopharmacology, 146: 447-454.

Björklund, A.; M. Jäntti and G. Solon (2007): Nature and Nurture in the Intergenerational Transmission of Socioeconomic Status: Evidence from Swedish Children and Their Biological and Rearing Parents. B. E. Journal of Advances in Economic Analysis and Policy, 7(2): Article 4.

Bouchard, T. J. (1998): Genetic and environmental influences on adult intelligence and special mental abilities. Human Biology, 70: 257-279.

Bouchard, T. J.; D. T. Lykken, M. McGue, M. Segal and N. L. Tellegen (1990): Sources of human psychological differences: The Minnesota study of twins reared apart. Science, 250: 223-228.

Brown, H. and M. van der Pol (2015): Intergenerational transfer of time and risk preferences. Journal of Economic Psychology, 49: 187-204.

Carpenter, J. P.; J. R. Garcia and J. K. Lum (2011): Dopamine receptor genes predict risk preferences, time preferences, and related economic choices. Journal of Risk and Uncertainty, 42(3): 233-261.

Carver, C. S. and C. J. Miller (2006): Relations of serotonin function to personality: Current views and a key methodological issue. Psychiatry Research, 144: 1-15.

Cesarini, D.; C. T. Dawes, J. Johannesson, P. Lichtenstein and B. Wallache (2009): Genetic Variation in Preferences for Giving and Risk Taking. Quaterly Journal of Economics, 124(2): 809-842.

Cronqvist, H. and S. Siegel (2015): The Origins of Savings Behavior. Journal of Political Economy, 123(1): 123-169. 
Dale, P. S.; M. G. Tosto, M. E. Hayiou-Thomas and R. Plomin (2015): Why does parental language input style predict child language development? A twin study of gene-environment correlation. Journal of Communication Disorders, 57: 106-117.

Dominicus, A.; A. Skrondal, H. K. Gjessing, N. L. Pedersen and J. Palmgren (2006): Likelihood ratio tests in behavioral genetics: Problems and solutions. Behavior Genetics, 36: 331-340.

Eisenberg, D.; J. MacKillop, M. Modi, J. Beauchemin, D. Dang, S. Lisman, J. Lum and D. Wilson (2007): Examining impulsivity as an endophenotype using a behavioral approach: A DRD2 TaqI A and DRD4 48-bp VNTR association study. Behavioral and Brain Functions, 3(2).

Fisher, R. A. (1918): The correlation between relatives on the supposition of Mendelian inheritance. Transactions of the Royal Society of Edinburgh, 52: 399-433.

Frederick, S.; G. Loewenstein and T. O'Donoghue (2002): Time Discounting and Time Preference: A Critical Review. Journal of Economic Literature, 40(2): 351-401.

Gauly, B. (2016): The Intergenerational Transmission of Attitudes: Analyzing Time Preferences and Reciprocity. Journal of Family and Economic Issues, doi:10.1007/s10834-016-9513-4.

Golsteyn, B. H.H.; H. Grönqvist and L. Lindahl (2014): Adolescent Time Preferences Predict Lifetime Outcomes. The Economic Journal, 124(158): 739-761.

Gouskova, E.; N. Chiteji and F. Stafford (2010): Pension Participation: Do Parents Transmit Time Preference? Journal of Family Economic Issues, 31: 138-150.

Hahn, E.; J. Gottschling, W. Bleidorn, C. Kandler, M. Spengler, A. E. Kornadt, W. Schulz, R. Schunck, T. Baier, K. Krell, V. Lang, F. Lenau, A. L. Peters, M. Diewald, R. Riemann and F. M. Spinath (2016): What Drives the Development of Social Inequality Over the Life Course? The German TwinLife Study. Twin Research and Human Genetics, 19(6): 659-672.

Holst, K. K. and T. Scheike (2017): Package 'mets': Analysis of Multivariate Event Times. R package version 1.2.1, https://cran.r-project.org/web/packages/ mets/index.html.

Kalenscher, T. and C. M. A. Pennartz (2008): Is a bird in the hand worth two in the future? The neuroeconomics of intertemporal decision-making. Progress in Neurobiology, 84: 284-315.

Kang, M.-I. and S. Ikeda (2014): Time dicounting and smoking behavior: Evidence from a panel survey. Health Economics, 23(12): 1443-1464. 
Knowles, J. and A. Postlewaite (2005): Do Children Learn to Save from Their Parents? Population Aging Research Centre (PARC) Working Paper Series, WPS 05-07, University of Pennsylvania.

Komlos, J.; P. K. Smith and B. Bogin (2004): Obesity and the rate of time preference: Is there a connection? Journal of Biosocial Science, 36(2): 209-219.

Kosse, F. and F. Pfeiffer (2012): Impatience among preschool children and their mothers. Economics Letters, 115(3): 493-495.

Maćkiewicz, M. and J. Cieciuch (2016): Pictorial Personality Traits Questionnaire for Children (PPTQ-C) - A New Measure of Children's Personality Traits. Frontiers in Psychology, 7(498): 1-11.

Meier, S. and C. Sprenger (2010): Present-Biased Preferences and Credit Card Borrowing. American Economic Journal: Applied Economics, 2(1): 193-210.

Mellor, D. and K. A. Moore (2014): The Use of Likert Scales with Children. Journal of Pediatric Psychology, 39(3): 369-379.

Miyazaki, K.; K. W. Miyazaki and K. Doya (2012): The Role of Serotonin in the Regulation of Patience and Impulsivity. Molecular Neurobiology, 45: 213-224.

Neale, M. C. and H. H. Maes (2004): Methodology for Genetic Studies of Twins and Families. Richmond, VA: Virginia Commonwealth University, Department of Psychiatry.

Oades, R. D; J. Lasky-Su, H. Christiansen, S. V. Faraone, E. J. S. Sonuga-Barke, T. Banaschewski, et al. (2008): The influence of serotonin- and other genes on impulsive behavioral aggression and cognitive impulsivity in children with attentiondeficit/hyperactivity disorder (ADHD): Findings from a family-based association test (FBAT) analysis. Behavioral and Brain Functions, 4(48).

Plomin, R.; J. C. Defries, V. S. Knopik and J. M. Neiderhiser (2013): Behavioral Genetics (6th ed.) New York, NY: Worth Publishers.

Rabe-Hesketh, S.; A. Skrondal and H. K. Gjessing (2008): Biometrical Modeling of Twin and Family Data Using Standard Mixed Model Software. Biometrics, 64: $280-288$.

Reynolds, B.; K. Leraas, C. Collins and S. Melanko (2004): Delay discounting by the children of smokers and non-smokers. Drug and Alcohol Dependence, 99: 350-353.

Rijsdijk, F. V. and P. C. Sham (2002): Analytic approaches to twin data using structural equation models. Briefings in Bioinformatics, 3(2): 119-133. 
Rossow, I. (2008): Alcohol consumption and discounting. Addiction Research and Theory, 16(6): 572-584.

Tsukayama, E. and A. L. Duckworth (2010): Domain-specific temporal discounting and temptation. Judgement and Decision Making, 5(2): 72-82.

Vischer, T.; T. J. Dohmen, A. Falk, D. Huffman, J. Schupp, U. Sunde and G. G. Wagner (2013): Validating an ultra-short survey measure of patience. Economics Letters, 120(2): 142-145.

Wagner, G. G.; J. R. Frick and J. Schupp (2007): The German Socio-Economic Panel Study (SOEP): Scope, Evolution and Enhancements. Schmollers Jahrbuch, 127(1): 139-169.

Waszczuk, M. A.; H. M. S. Zavos, E. Antonova, C. M. Haworth, R. Plomin and T. C. Eley (2015): A multivariate twin study of trait mindfulness, depressive symptoms, and anxiety sensitivity. Depression and Anxiety, 32: 254-261.

Webley, P. and E. K. Nyhus (2006): Parents' influence on children's future orientation and saving. Journal of Economic Psychology, 27: 140-164.

Zhong, S.; S. H. Chew, E. Set, J. Zhang, H. Xue, P. C. Sham, R. P. Ebstein and S. Israel (2009): The Heritability of Attitude Toward Economic Risk. Twin Research and Human Genetics, 12(1): 103-107.

Zyphur, M. J.; J. Narayanan, R. D. Arvey and G. J. Alexander (2009): The Genetics of Economic Risk Preferences. Journal of Behavioral Decision Making, 22: 367-377. 


\section{Figures in text}

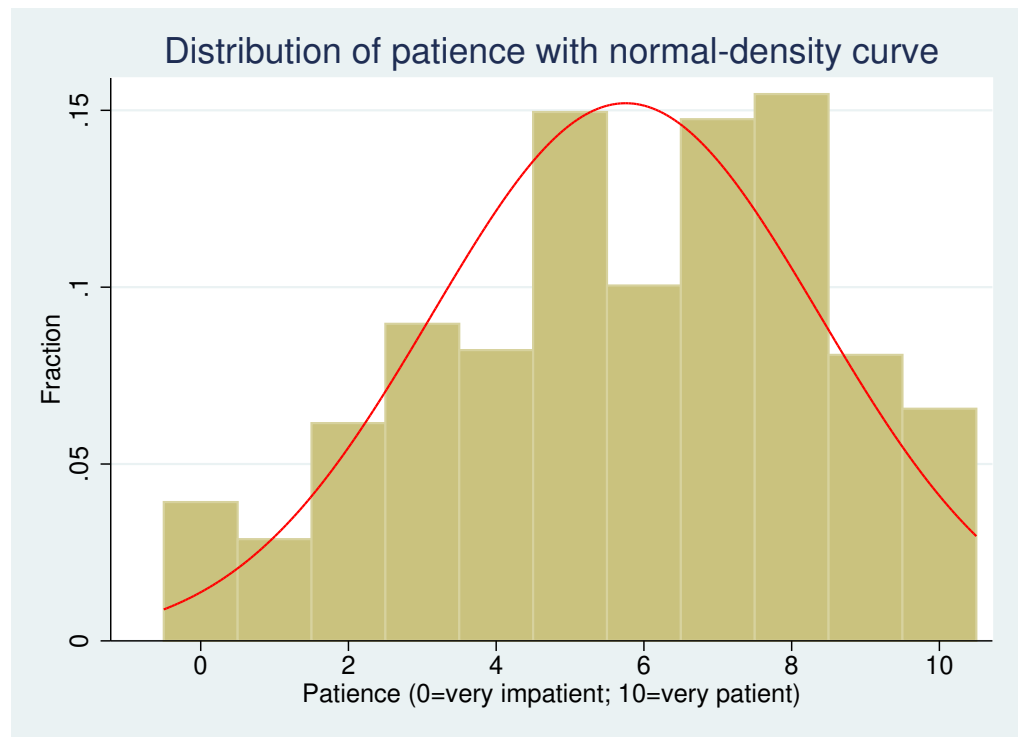

Figure 1: Distribution of patience with the plot of a normal-density curve

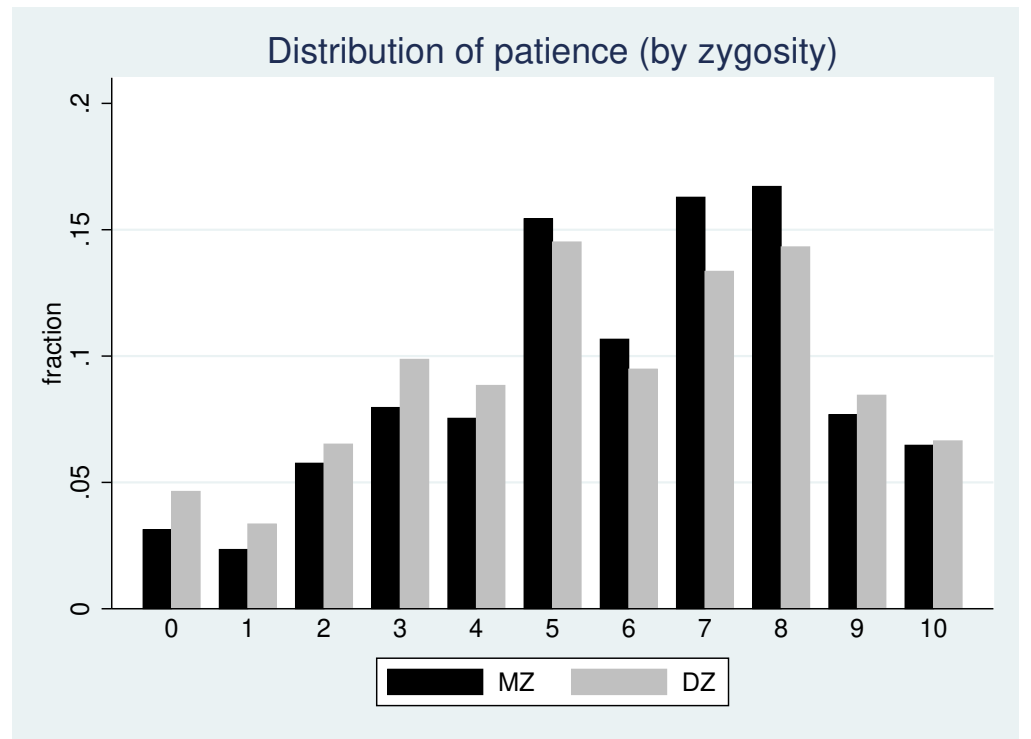

Figure 2: Distribution of patience, by zygosity 


\section{Twin-twin scatterplots (by zygosity)}

MZ twins (\# pairs: 703)

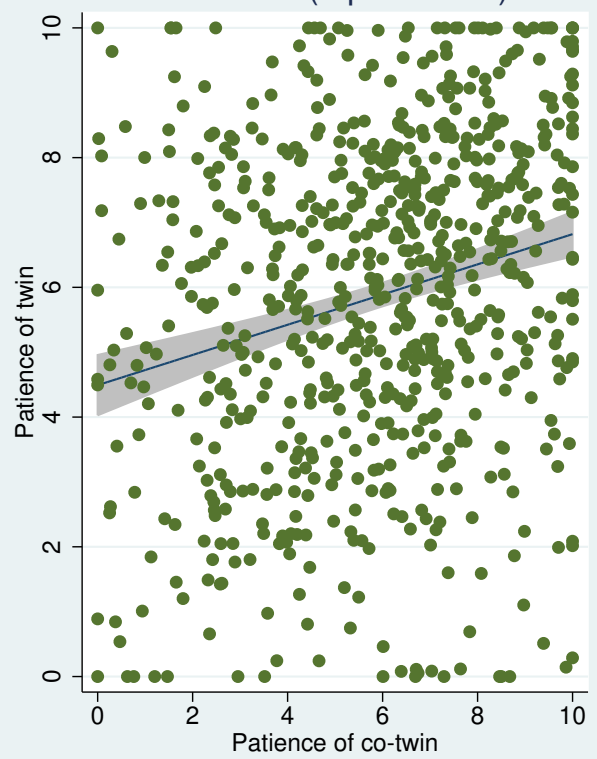

DZ twins (\# pairs: 775)

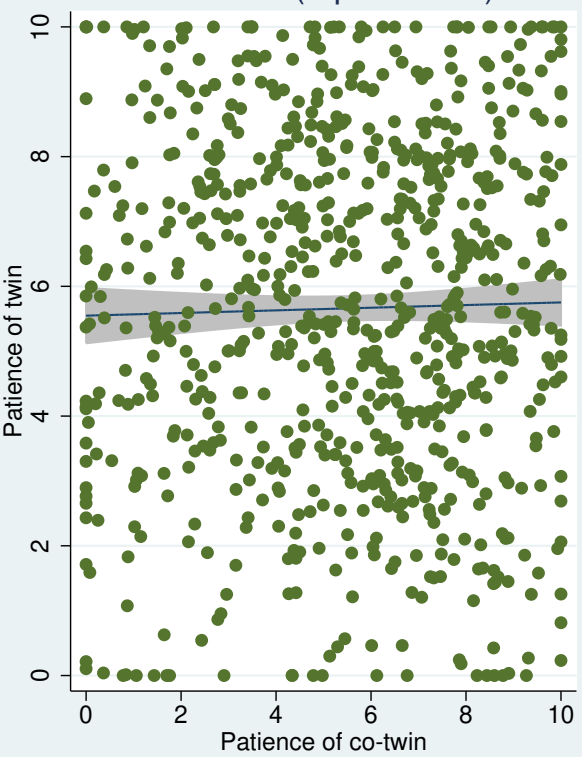

Figure 3: Twin-twin plot of patience, by zygosity (Plots include fitted regression line and $95 \%$ conficence intervals.) 
Tables in text

Table 1: Number of twins by zygosity and gender

\begin{tabular}{l|c|c}
\hline \hline & female & male \\
\hline DZ twins & 856 & 694 \\
MZ twins & 806 & 600 \\
\hline
\end{tabular}

Table 2: Summary statistics of the final twin sample $(\mathrm{n}=2956)$

\begin{tabular}{lcccc}
\hline \hline Variable & Mean & S. D. & Min. & Max. \\
\hline patience & 5.753 & 2.624 & 0 & 10 \\
female & 0.562 & 0.496 & 0 & 1 \\
age & 17.095 & 5.085 & 10 & 25 \\
MZ & 0.476 & 0.499 & 0 & 1 \\
\hline
\end{tabular}


Table 3: Self-reported patience

\begin{tabular}{llccc}
\hline \hline & & MZ twins & DZ twins & p-value \\
\hline Patience & Mean & 5.907 & 5.614 & $<.01$ \\
& S.D. & 2.522 & 2.707 & \\
& n & 1406 & 1550 & \\
\hline
\end{tabular}

Notes: p-value from two-sample t test. Null hypothesis:

Same mean of patience for MZ and DZ twins.

Table 4: Correlations for MZ and DZ twin pairs

\begin{tabular}{|c|c|c|c|}
\hline & MZ twin pairs & DZ twin pairs & $\begin{array}{l}\text { p-value } \\
\text { of diff. }\end{array}$ \\
\hline Patience Spearman & $\begin{array}{c}0.241^{* * *} \\
(0.170-0.310)\end{array}$ & $\begin{array}{c}0.006 \\
(-0.064-0.077)\end{array}$ & $<.01$ \\
\hline $\mathrm{n}$ & 703 & 775 & \\
\hline \multicolumn{4}{|c|}{$\begin{array}{l}\text { Notes: Significance: }{ }^{*} \mathrm{p}<0.1,{ }^{* *} \mathrm{p}<0.05,{ }^{* * *} \mathrm{p}<0.01 \text {. Confidence inter- } \\
\text { vals for Spearman's rank correlations in parentheses. p-value from boot- } \\
\text { strapped equality test for equal correlation coefficients. Null hypothesis: } \\
\text { Same correlations of patience for MZ and DZ twin pairs. Bootstrapped } \\
\text { replications }=1000 \text {. }\end{array}$} \\
\hline
\end{tabular}


Table 5: Results of the biometric analyis of patience

\begin{tabular}{lccccccc|cccc}
\hline \hline & $\mathrm{LL}$ & $\mathrm{df}$ & $\mathrm{LRT}$ & $\Delta \mathrm{df}$ & $\mathrm{p}$-value & $\mathrm{AIC}$ & $\mathrm{BIC}$ & $\mathrm{A}$ & $\mathrm{D}$ & $\mathrm{C}$ & $\mathrm{E}$ \\
\hline $\begin{array}{l}\text { Saturated model } \\
\text { ADE }\end{array}$ & $-4155,544$ & 18 & & & & 8347,088 & 8442,46 & & & & \\
& $-4167,294$ & 6 & 23.499 & 12 & $0.024 \dagger$ & 8346,588 & 8378,378 & 0.000 & 0.234 & - & 0.766 \\
$\mathrm{AE}$ & $-4170,332$ & 5 & 6.077 & 1 & $<.01 \ddagger$ & 8350,664 & 8377,156 & $(0.000-0.000)$ & $(0.162-0.306)$ & $(0.694-0.838)$ \\
& & & & & & & & $(0.194$ & - & - & 0.806 \\
$\mathrm{DE}$ & $-4167,294$ & 5 & $<.01$ & 1 & $>.10 \ddagger$ & 8344,588 & 8371,08 & - & $0.261)$ & & $(0.739-0.873)$ \\
& & & & & & & & & $(0.162-0.306)$ & $(0.694-0.838)$ \\
\hline
\end{tabular}

Notes: Results from the variance component analysis. $\mathrm{LL}=\log$ Likelihood, $\mathrm{df}=$ degrees of freedom, $\mathrm{LRT}=$ Likelihood ratio test statistic, $\Delta \mathrm{df}=$ difference in degrees of freedom, $\mathrm{AIC}=$ Akaike's information criterion, BIC=Sample size adjusted Bayesian's information criterion. $\dagger$ Compared to the saturated model. $\ddagger$ Compared to the ADE model. Adjusted p-values (Dominicus et al. 2006). 95\% confidence intervals in parentheses. 